\title{
THE RHEOLOGICAL BEHAVIOR OF DISPERSE SYSTEMS FOR 3D PRINTING IN CONSTRUCTION: THE PROBLEM OF CONTROL AND POSSIBILITY OF «NANO» TOOLS APPLICATION
}

\section{Extended Abstract:}

The paper considers the problem of creating a wider class of building materials used for 3D printing. From the point of view of classical rheology of disperse systems, the application of 3D printing technology in construction has been analyzed. Theoretical analysis of the models of rheological behavior is performed according to state of their structure and the dynamic of the $3 \mathrm{D}$ printing processes such as mixing, pumping, extrusion, multilayer casting and structural built-up in the printing layers. The main factors and criteria for the stability of heterogeneous disperse systems in dynamic and static 3D printing processes have been identified. The general scientific concept for optimization of admixtures for 3D printable materials has been developed in terms of viscosity, consistency, and parameters of flocculation and structural built-up. The technological tools to control rheological behavior of visco-plastic admixtures are identified in all stages of 3D printing. The relevant considerations include the concentration, size, morphology, chemical and mineralogical composition, the physical and chemical activity of the solid phase's surface, and the ionic composition, viscosity, and density of the liquid phase. It is shown that the practical engineering solutions to control the rheology, structure formation, properties of $3 \mathrm{D}$ printing admixtures and materials must be based on traditional factors as well as with the use of «nano» tools. According to the nanotechnological principle «bottom-up», a set of 
«nano» tools is proposed to control such admixture properties as extrudability, formability, and buildability. In conclusion the scientific and technical tasks to be researched have been formulated.

Key words: disperse systems, structural and rheological properties, rheological behavior of admixtures, $3 \mathrm{D}$ printing in constrcution.

DOI: dx.doi.org/10.15828/2075-8545-2018-10-3-107-122

\footnotetext{
Machine-Readable information on CC-licenses (HTML-code) in Metadata of The PAPER

$<$ a rel="license" href="http://creativecommons.org/licenses/by/4.0/" > <img alt="Creative Commons License" style="borderwidth:0" src="https://i.creativecommons.org/l/by/4.0/88x31.png" $/></ \mathrm{a}><$ br $/><$ span xmlns:dct="http://purl.

org/dc/terms/" property="dct:title" $>$ The rheological behavior of disperse systems for 3D print-ing in constrcution: the problem of control and possibility of «nano» tools application </span $>$ by < a xmlns:cc="http://creativecommons.org/ns\#" href="Nanotehnologii v stroitel'stve = Nanotechnologies in Construction. 2018, Vol. 10, no. 3, pp. 107-122. DOI: dx.doi. org/10.15828/2075-8545-2018-10-3-107-122" property="cc:attributionName" rel="cc:attributionURL">Slavcheva G.S., Artamonova O.V. $</$ a $>$ is licensed under a $<$ a rel="license" href="http://creativecommons.org/licenses/by/4.0/" $>$ Creative Commons Attribution 4.0 International License $</ \mathrm{a}>$. $<$ br $/>$ Based on a work at $<$ a xmlns:dct="http: //purl.org/dc/terms/" href="http://nanobuild.ru/en_EN/nanobuild-3-2018/" rel="dct:source" $>$ http://nanobuild.ru/en_EN/nanobuild-3-2018/</ a $>$. $<$ br $/>$ Permissions beyond the scope of this license may be available at $<$ a xmlns:cc="http://creativecommons.org/ns \#" href="ol_artam@rambler.ru"rel="cc:morePermissions">ol_artam@rambler.ru</a $>$.
}

\section{References:}

1. Lim S., Buswell R.A., Le T.T., et al. Developments in construction-scale additive manufacturing processes.Automation in Construction. 2012. 21 (1). Pp. 262-268.

2. Gebler M., Schoot Uiterkamp A.J.M., \& Visser C. A global sustainability perspective on 3D printing technologies. Energy Policy. 2014. 74(C). Pp. 158-167.

3. Tay Y.W., Panda B., Paul S.C., et al. Processing and Properties of Construction Materials for 3D Printing. Materials Science Forum. 2016. 861. Pp. 177-181.

4. Lloret E., Shahabb A.R., Linus M., et al. Complex concrete structures Merging existing casting techniques with digital fabrication. Computer-Aided Design. 2015. V. 60. Pp. 40-49.

5. Weng Y., Li M., Qian S., et al. Design 3D printing cementitious materials via Fuller Thompson theory and Marson-Percy model. Construction and Building Materials. 2018. V. 163. Pp. 600-610. 
6. Roussel $N$. Steady and transient flow behaviour of fresh cement pastes. Cement and Concrete Research. 2005. 35 (9). Pp. 1656-1664.

7. Khalil N., Aouad G., El Cheikh K.\& Rémond S. Use of calcium sulfoaluminate cements for setting control of 3D-printing mortars. Construction and Building Materials. 2017. V. 157. Pp. 382-391.

8. Panda B., \& Tan M.J. Experimental study on mix proportion and fresh properties of fly ash based geopolymer for 3D concrete printing. Ceramics International. 2018. V. 44. Is. 9. Pp. 10258-10265.

9. Paul S.C., Tay Y.W.D., Panda B., \& Tan M.J. Fresh and hardened properties of 3D printa-ble cementitious materials for building and construction. Archives of Civil and Mechanical Engi-neering. 2018. V. 18(1). Pp. 311-319.

10. Van Zijl A., Chandra S., Tan M.J., et al. Properties of 3D Printable Concrete. $2^{\text {nd }}$ Interna-tional Conference on Progress in Additive Manufacturing, (Pro-AM 2016). At Nanyang, Singapore, 2016. Pp. 421-426.

11. Perrot A., Rangeard D. \& Pierre A. Structural built-up of cement-based materials used for 3D-printing extrusion techniques. Materials and Structures. 2016. V. 49. Pp. 1213-1220.

12. Chernyshev E.M., Artamonova O.V., Slavcheva G.S. Concepts and technology base nano-modification of structures of building composites. Part 3: effective nanomodification of systems and structures of cement hardening cement stone (criteria and conditions). Construction materials. 2015. № 10. Pp. 54-63. (In Russian).

13. Chernyshev E.M., Artamonova O.V., Slavcheva G.S. Nanomodificirovanie sistem tverdeniya $\mathrm{v}$ strukture stroitel'nyh kompozitov [Nanomodification of hardening systems in the struc-ture of building composites]. Voronezh, Nauchnaya kniga [The scientific book]. 2016. 132 p. (In Russian).

14. Chernishov E.M., Slavcheva G.S., Artamonova O.V. Control of rheological and structural properties cement stone at the nanomodification. Nanotehnologii v stroitel'stve $=$ Nanotechnologies in Construction. 2016, Vol. 8, no. 6, pp. 87-101. DOI: dx.doi.org/10.15828/2075-8545-2016-8-6-87-101. (In Russian).

15. Rebinder P.A. Izbrannye trudy. Poverhnostnye yavleniya $\mathrm{v}$ dispersnyh sistemah [Selected works. Surface phenomena in disperse systems. Physico-chemical mechanics]. Moscow: Nauka. 1979. 381 p. (In Russian).

16. Kruglitsky N.N. Ocherki po fiziko-himicheskoj mekhanike [Essays on physical and chem-ical mechanics]. Kiev, Naukova Dumka, 1988. 224 p. (In Russian).

17. Uryev N.B. Fiziko-himicheskaya dinamika strukturirovannyh nanodispersnyh sistem i nanodispersnyh kompozicionnyh materialov. Chast' 1 [Physico-chemical dynamics of structured nanodispersed systems and nanodispersed composite ma- 
terials. Part 1]. Fizikohimiya poverhnosti i zashchita materialov. [Physicochemistry of the surface and protection of materials]. 2010. Vol. 46, № 1. Pp. 3-23. (In Russian).

18. Uryeu N.B. Vysokokoncentrirovannye dispersnye sistemy [Highly concentrated disperse systems]. Moscow: Himiya [Chemistry], 1980. 319 p. (In Russian).

19. Yaminsky V.V., Pchelin V.A., Amelina E.A., Shchukin E.D. Koagulyacionnye kontakty v dispersnyh sistemah [Coagulation contacts in disperse systems]. Moscow: Himiya [Chemistry]. 1982. 185 p. (In Russian).

20. Koch C.C. Nanostructured materials: Processing, Properties and Potential Applications. New York: Noyes Publications, 2002.612 p.

\section{DeAR Colleagues!}

THE REFERENCE TO THIS PAPER HAS THE FOLLOWING CITATION FORMAT:

Slavcheva G.S., Artamonova O.V. The rheological behavior of disperse systems for 3D print-ing in constrcution: the problem of control and possibility of «nano" tools application. Nanotehnologii v stroitel'stve $=$ Nanotechnologies in Construction. 2018, Vol. 10, no. 3, pp. 107-122. DOI: dx.doi.org/10.15828/2075-85452018-10-3-107-122. (In Russian). 
Автор: СЛАВЧЕВА Галина Станиславовна, д.т.н., проф. каф. технологии строительных материалов, изделий и конструкций, Воронежский государственный технический университет; ул. 20-летия Октября, 84, Воронеж, 394006, e-mail: gslavcheva@yandex.ru;

Автор: АРТАМОНОВА Ольга Владимировна, к.х.н., доц. каф. химии и химической технологии материалов, Воронежский государственный технический университет; ул. 20-летия Октября, 84, Воронеж, 394006, e-mail: ol_artam@rambler.ru

\section{РЕОЛОГИЧЕСКОЕ ПОВЕДЕНИЕ ДИСПЕРСНЫХ СИСТЕМ ДЛЯ СТРОИТЕЛЬНОЙ ЗD-ПЕЧАТИ: ПРОБЛЕМА УПРАВЛЕНИЯ НА ОСНОВЕ ВОЗМОЖНОСТЕЙ АРСЕНАЛА «НАНО»}

\section{АННОТАЦИЯ К СТАТЬЕ (АВТоРСКОЕ РЕЗЮме, РЕФЕРАТ):}

В статье обсуждается проблема создания смесей и композитов широкого функционального назначения, адаптированных к процессам строительной 3D-печати. Исходя из положений классической реологии дисперсных систем, проанализирован строительно-технологический процесс 3D-печати. Произведен теоретический анализ моделей реологического поведения дисперсных систем в соответствии с состоянием их структуры и динамикой процессов приготовления смеси, транспортирования ее из смесителя в экструдер, экструдирования, послойной укладки по заданной траектории, твердения смесей в напечатанных слоях строительного объекта. Выделены основные факторы и критерии устойчивости гетерогенных дисперсных систем в динамических и статических условиях 3D-печати. Сформирована общая научная концепция оптимизации составов смесей для 3D-печати по характеристикам вязкости, пластичности, а также параметрам процессов схватывания и твердения. Обоснованы технологические средства управления реологическим поведением вязко-пластичных смесей на всех стадиях процесса 3D-печати. К ним отнесены концентрация, размер, морфология, химико-минералогический состав, физико-химические свойства поверхности частиц дисперсной фазы, ионный состав, вязкость, плотность дисперсионной среды. Показано, что прикладные инженерные решения по управлению реологией, структурообразованием 
и свойствами смесей и композитов для 3D-печати должны быть основаны на применении традиционных рецептурно-технологических факторов и использовании средств из арсенала «нано». В работе предложен набор средств из арсенала «нано» в соответствии с нанотехнологическим принципом «снизу вверх", в частности, для управления реологическими характеристиками смесей, для обеспечения их вязкопластичности и формоустойчивости. В заключении сформулированы научные и прикладные задачи дальнейшего развития исследований в этой области.

Ключевые слова: дисперсные системы, структурно-реологические свойства, реологическое поведение смесей, 3D-печать в строительстве.

DOI: dx.doi.org/10.15828/2075-8545-2018-10-3-107-122

МАШИНОЧИТАЕМАЯ ИНФОРМАЦИЯ О СС-ЛИЦЕНЗИИ В МЕТАДАННЫХ СТАТЬИ (НTML-КОД):

$<$ a rel="license" href="http://creativecommons.org/licenses/by/4.0/"><img alt="Лицензия Creative Commons" style="borderwidth:0" src="https://i.creativecommons.org/l/by/4.0/88x31.png" / $></ \mathrm{a}><$ br $/>$ Произведение «<span xmlns:dct="http:// purl.org/dc/terms/" href="http://purl.org/dc/dcmitype/Text" property="dct:title" rel="dct:type"> Реологическое поведение дисперсных систем для строительной $3 \mathrm{~d}-$-печати: проблема управления и возможности арсенала «нано» </span>» созданное автором по имени <a xmlns:cc="http://creativecommons.org/ns\#" href="Нанотехнологии в строительстве. $-2018 .-$ Том 10, № 3. - C. 107-122. - DOI: dx.doi.org/10.15828/2075-8545-2018-10-3-107-122." property="cc:attributionName" rel="cc:attribut ionURL"> Славчева Г.С., Артамонова О.В. </a>, публикуется на условиях < a rel="license" href="http://creativecommons.org/ licenses/by/4.0/">лицензии Creative Commons C указанием авторства 4.0 Всемирная $</ \mathrm{a}>$. $<\mathrm{br} />$ Основано на произведении c < a xmlns:dct="http://purl.org/dc/terms/" href="http://nanobuild.ru/ru_RU/nanobuild-3-2018/" rel="dct:source">http:// nanobuild.ru/ru_RU/nanobuild-3-2018/</a >. $<$ br / Pазрешения, выходящие за рамки данной лицензии, могут быть доступны на странице $<$ a xmlns:cc="http://creativecommons.org/ns\#" href="ol_artam@rambler.ru" rel="cc:morePermissions" $>$ ol_ artam@rambler.ru</a $>$.

\section{Введение}

Применение нанотехнологических принципов и подходов в строительном материаловедении обеспечило качественно новые возможности управления структурой и свойствами материалов. Использование средств из арсенала «нано» (в соответствии с терминологией, введенной академиком РАACH E.M. Чернышовым) позволило получить строительные композиты нового поколения с уникальным комплексом свойств. Однако данные высокотехнологичные композиты, получаемые с применением микро- и наноразмерных структурных составля- 
ющих, не находят широкого применения в строительстве. Сложилось противоречие между достижениями и технологическими возможностями материаловедения и нанотехнологий и потребностями традиционных технологий производства материалов для массового строительства. Разработанные концепции и средства из арсенала «нано» могут быть востребованы для реализации именно тех строительно-технологических процессов, которые основаны на новых нетрадиционных технологических принципах и приемах. На наш взгляд, к ним следует отнести строительные 3D-аддитивные технологии. Именно для их реализации в строительно-технологической практике необходим накопленный потенциал и арсенал «нано» .

Как известно, отличительной особенностью процесса создания любых объектов с помощью 3D-аддитивных методов является использование цифровых технологий. Процесс реализуется через создание цифровой 3D-модели объекта; "разрезание» ее на 2D-слои с использованием соответствующего программного обеспечения, программирование работы исполнительного механизма для последовательного нанесения слоев материала в соответствии с его 3D-моделью. В настоящее время для создания строительных объектов апробированы и развиваются следующие 3D-аддитивные технологии, отличающиеся принципами формирования структуры объекта [1-3]: 3D-печать - объект создается при послойном экструдировании вязко-пластичной смеси, D-Shape - объект создается при послойном нанесении наполнителя и жидкого связующего, Slipforming - объект создается при экструдировании смеси в момент ее схватывания. Наиболее приемлемой для практики строительства оказалась 3D-печать, строительно-технологический процесс реализации которой включает стадии приготовления смеси, транспортирования ее из смесителя в экструдер, экструдирование, послойную укладку, твердение смесей. Аппаратурное оформление данного способа доведено до промышленного выпуска комплектов оборудования рядом российских и зарубежных фирм.

\section{Постановка проблемы}

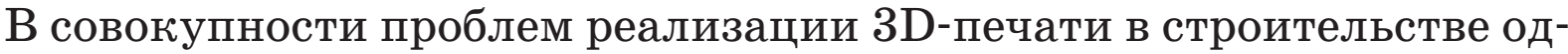
ной из наиболее сложных и комплексных является проблема создания смесей. Несмотря на активное развитие исследований и большой объ- 
ем накопленной экспериментальной информации [4-11], следует отметить, что на сегодня отсутствует системное представление о принципах и приемах управления структурно-реологическими характеристиками смесей, кинетикой процессов схватывания и твердения.

Анализ процесса 3D-печати позволяет структурировать проблему создания адаптированных к данному процессу смесей на следующие составляющие: 1) проблема регулирования вязкости и пластичности смеси для обеспечения ее подачи к соплу принтера и экструзии; 2) проблема регулирования формоустойчивости и прочности смеси для обеспечения ее послойной укладки без деформирования; 3) проблема регулирования кинетики структурообразования и твердения композита для синхронизации скорости набора прочности со скоростью печати строительного объекта; 4) проблема оптимизации составов смесей по характеристикам вязкости, пластичности, скорости твердения и прочности для композитов различного функционального назначения и области применения в строительстве.

Для решения данной проблемы необходимо формирование общей научной концепции и обоснования комплексных технологических решений по созданию композитов широкого функционального назначения, адаптированных к процессам строительной 3D-печати. Возможности арсенала «нано» для организации кинетики структурообразования, модифицирования структуры и свойств были теоретически обоснованы и показаны в наших работах [12-14]. В данной статье акцент делается на выборе средств из арсенала «нано» для управления реологическими характеристиками смесей.

\section{Теоретическое обоснование концепции управления реологическим поведением дисперсных систем для 3D-печати}

В соответствии с фундаментальными положениями структурной реологии (работы школ П.А Ребиндера [15] и А.В. Думанского [16]) дисперсные системы в технологическом процессе 3D-печати последовательно проходят целый спектр состояний структуры, которые соотносятся с его стадиями следующим образом: 1) приготовление смеси - формирование высококонцентрированной дисперсной системы «твердая дисперсная фраза - жидкая дисперсионная среда», структу- 
рированной коагуляционными контактами между частицами дисперсной фазы; 2) транспортировка смеси - вязко-пластическое течение при действии высоких напряжений сдвига, система переходит в состояние с полностью разрушенной структурой; 3) экструзия - вязкопластическое течение при действии низких сжимающих напряжений, в системе начинается процесс восстановления коагуляционных контактов; 4) печать слоя - переход в твердообразное состояние и восстановление коагуляционной структуры; 5) схватывание и твердение напечатанных слоев, система переходит от вязко-пластичной коагуляционной структуры к формированию структуры твердого тела с коагуляционно-кристаллизационными фазовыми контактами в результате химических и фазовых превращений.

На стадии существования коагуляционной структуры реологическое поведение дисперсных систем «твердая дисперсная фаза - жидкая дисперсионная среда» определяется действием следующего баланса сил: поверхностных сил молекулярного притяжения $f_{c}$ и электростатического отталкивания $f_{\mu}$, определяющих поверхностные явления и межчастичные взаимодействия; внутренней энергии колебаний молекул дисперсионной среды, ответственных за тепловое броуновское движение; внешних сил, определяющих инерционность и гидродинамическое движение частиц в потоке; силы тяжести, влияющей на процесс седиментации частиц.

Вклад каждой из составляющих данного баланса сил зависит от динамики внешних воздействий и от типа дисперсной системы. В процессе 3D-печати смеси после приготовления при перекачке и экструзии находятся в динамических условиях, в процессе укладки слоев - в статических. Как правило, для реализации данных процессов используют две группы смесей, которые, согласно [17], можно квалифицировать как грубодисперсные (размер частиц $d>100$ мкм) и микродисперсные (размер частиц $d \sim 1 \div 100$ мкм). Для таких систем вклад броуновского движения является пренебрежимо малым, а вклад поверхностных явлений, межчастичных взаимодействий, инерционность, гидродинамический фактор и фактор седиментации - главенствующими [18].

В динамических условиях поведение таких систем определяется кинетической энергией внешних сил, динамикой столкновения (соударения) соседних частиц и гидродинамическим сопротивлением дисперсионной среды, действием силы тяжести. Данные силы вызывают инер- 
ционные и гидродинамические эффекты, сопровождающими движение частиц в пространстве. В микродисперсных системах с сильно развитой межфазной поверхностью существенную роль играют контактные взаимодействия между частицами, а также совокупность поверхностных явлений на межфазных границах [19-20]. В статических условиях поведение гетерогенных грубо- и микродисперсных систем определяется действием сил тяжести (фактор седиментации) и силами внутренних взаимодействий (вклад поверхностных явлений, контактных взаимодействий). Частицы объединяются в пространственные структуры, если поле действия данных сил соизмеримо с силой тяжести.

Анализ классических положений структурной реологии позволил выделить основные факторы устойчивости рассматриваемых систем в динамических и статических условиях. К ним отнесены: 1) кинетический, связанный со способностью дисперсной фазы находиться во взвешенном состоянии в дисперсионной среде и противодействовать кинетической энергии внешних сил и силам тяжести. Для этого необходимо сохранение определенного критического размера и уровня свободной поверхностной энергии частиц дисперсной фазы; 2) электростатический, который способствует взаимному отталкиванию дисперсных частиц из-за существования на их поверхности двойного электрического слоя. Его реализация зависит от наличия одноимённого заряда частиц дисперсной фазы, значения их электрокинетического потенциала, а также изменения межфазного поверхностного натяжения вследствие адсорбции ионов электролитов; 3) молекулярно-адсорбционный, связанный с условиями, которые также препятствуют агрегации частиц дисперсной фазы. Его реализации способствует снижение уровня свободной межфазной энергии на границе раздела фаз, уменьшение силы взаимодействия в контактах между частицами. Средствами реализации являются гидратация (сольватация) частиц дисперсной фазы, а также модификация их поверхности защитным слоем из адсорбированных молекул ПАВ; 4) гидродинамический, определяющий зависимость устойчивости дисперсных систем от плотности и вязкости дисперсионной среды. При их увеличении снижается подвижность частиц дисперсной фазы в дисперсионной среде и возрастает устойчивость системы.

Поведение грубо- и микродисперсных систем в динамических и статических условиях процесса 3D-печати лимитируется взаимодействием 
этих факторов. Результативность их влияния на устойчивость систем оценивается двумя критериями - агрегируемостью и прочностью, теоретически обоснованными в работах [17-19].

\section{Критерий агрегируемости}

Коагуляционная структура в дисперсных системах может возникнуть и сохранять устойчивость, если силы сцепления $f_{c}$ между частицами соизмеримы или превышают вес частиц $G^{*}$ в дисперсионной среде [17]. Вес частиц определяется из соотношения

$$
G^{*}=\rho_{e f f} d^{3} g
$$

где $\rho_{\text {eff }}-$ приведенная плотность частицы в дисперсионной среде ( $\rho_{e f f}=\rho_{0}-\rho_{1}, \rho_{0}-$ плотность твердой фазы, $\rho_{1}-$ плотность дисперсионной среды), $d$ - характерный размер частицы, $g$ - ускорение силы тяжести.

Получено соотношение для диапазона критических размеров частиц dc, которые могут образовать устойчивые коагуляционные структуры [17]:

$$
\frac{d_{\mathrm{c}}}{2}=r_{\mathrm{c}} \equiv\left[\left(U_{i} / r\right) /\left(\frac{4}{3} \pi \rho_{e f f} g h_{i}\right)\right]^{1 / 2},
$$

где $U_{i}$ - энергия взаимодействия частиц в контакте, $h_{i}-$ расстояние между частицами.

Найденные в [17] численные значения $d_{c}$ показали, что самопроизвольное образование коагуляционных структур возможно в дисперсиях с размером частиц в десятки и даже сотни микрометров. Применительно к смесям для 3D-печати, которые как высококонцентрированные пасты относятся к дисперсным системам с ближней коагуляцией частиц, величина $d_{c} / 2 \sim 5 \times 10^{-5}-10^{-4} \mathrm{M}$.

Критерий прочности определяет функциональную зависимость прочности структуры дисперсной системы от размера частиц (в области $<d_{c}$ ), прочности индивидуальных контактов и их числа в единице объема структуры, зависящего от концентрации дисперсной фазы $\varphi$ в дисперсионной среде [17]: 


$$
P_{m}=\alpha_{i} F_{c} n^{2 / 3}=\alpha_{i} F_{c} f(\varphi) / \overline{d^{2}},
$$

где $P_{m}-$ прочность структуры, $\alpha_{i}-$ коэффициент, характеризующий тип упаковки частиц, $F_{c}$ - сила сцепления в контакте, $n$ - число контактов между частицами в единице объема, $\varphi$ - объемная концентрация частиц, $d-$ их средний размер.

Доказано [17], что критические концентрации частиц дисперсной фазы $\varphi_{0}$ (образования коагуляционной структуры) и $\varphi_{c}$ (ее упрочнения) для каждой пары «твердая дисперсная фаза - жидкая дисперсионная среда» зависят от характеристик частиц дисперсной фазы и свойств дисперсионной среды.

Исходя из анализа факторов и критериев устойчивости и прочности дисперсных систем на стадии существования коагуляционной структуры, можно выделить следующие средства управления их реологическим поведением в статических и динамических условиях 3D-печати:

1) по отношению к дисперсной фазе - ее концентрация $\varphi$, размер частиц $d_{c}$ и их морфология, химико-минералогический состав, физико-химические свойства поверхности частиц, определяющие вклад кинетического, электростатического и молекулярно-адсорбционного факторов;

2) по отношению к дисперсионной среде - ее ионный состав, вязкость, плотность, определяющие вклад электростатического, молекулярно-адсорбционного и гидродинамического факторов.

\section{Концепция реализации возможностей арсенала «нано» для управления реологическим поведением дисперсных систем в условиях строительной 3D-печати}

Речь идет о выборе традиционных рецептурно-технологических средств и средств из арсенала «нано» для решения задач:

1) оптимизации реологических характеристик смесей (вязкопластичности $\eta$, формоустойчивости $F$ ) при обеспечении задаваемого уровня качества получаемых композитов $\bar{R}$.

2) организации кинетики процессов схватывания и твердения (скорости и времени завершения $\tau$ ).

В наших исследованиях $[12,13]$ относительно решения второй задачи к возможностям арсенала «нано» были отнесены технологии и тех- 
ники наноструктурирования и (или) наномодифицирования строительных композитов по принципу «сверху - вниз» и «снизу - вверх». Для решения первой задачи обоснование факторного пространства управления (табл.) основано на представленных выше средствах повышения устойчивости дисперсных систем на стадии формирования коагуляционной структуры.

таблииа

\section{Средства и рецептурно-технологические факторы регулирования реологических характеристик смесей для 3D-печати}

\begin{tabular}{|c|c|c|}
\hline $\begin{array}{l}\text { Компоненты } \\
\text { смеси }\end{array}$ & $\begin{array}{c}\text { Средства } \\
\text { регулирования }\end{array}$ & $\begin{array}{c}\text { Рецептурно-технологические факторы } \\
\text { управления }\end{array}$ \\
\hline \multirow{5}{*}{$\begin{array}{l}\text { дисперсная } \\
\text { фаза }\end{array}$} & концентрация & состав смеси по В/Т-отношению \\
\hline & размер частиц & $\begin{array}{l}\text { гранулометрия наполнителей и заполни- } \\
\text { телей }\end{array}$ \\
\hline & морфология частиц & $\begin{array}{l}\text { соотношение } d / l \text { частиц наполнителей } \\
\text { и заполнителей }\end{array}$ \\
\hline & $\begin{array}{l}\text { химико-минералоги- } \\
\text { ческий состав }\end{array}$ & $\begin{array}{l}\text { состав смеси по C/S - вид связующего, } \\
\text { наполнителей и заполнителей }\end{array}$ \\
\hline & $\begin{array}{l}\text { свойства поверхности } \\
\text { частиц }\end{array}$ & $\begin{array}{l}\text { условия и режимы диспергации (помо- } \\
\text { ла); условия и режимы перемешивания; } \\
\text { виды и дозировки добавок ПАВ }\end{array}$ \\
\hline \multirow[b]{2}{*}{$\begin{array}{l}\text { дисперсионная } \\
\text { среда }\end{array}$} & ионный состав & виды и дозировки добавок электролитов \\
\hline & вязкость / плотность & $\begin{array}{l}\text { температура, виды и дозировки добавок } \\
\text { модификаторов вязкости (коагулянтов) } \\
\text { и наномодификаторов }\end{array}$ \\
\hline
\end{tabular}

Общими для решения обеих задач являются факторы варьирования состава смеси по В/Т-отношению, виду и характеристикам связующего и наполнителей, условий и режимов диспергации (помола) и перемешивания, температуре твердения, вида и дозировок добавок ПАВ. При этом необходимо учитывать и дополнительные специальные факторы для управления реологией, а именно варьирование соотношения $d / l$ частиц наполнителей и заполнителей, использование добавок электролитов, модификаторов вязкости (коагулянтов) и наномодификаторов. 
Из всей совокупности факторов управления реологическим поведением дисперсных систем в процессе 3D-печати к традиционным рецептурно-технологическим факторам относятся химико-минералогический состав и гранулометрия связующего, заполнителей и наполнителей, механохимическая активация поверхности при помоле и перемешивании, температура как средство регулирования растворимости компонентов сырьевой смеси и соответственно скорости и степени насыщения системы катионами и анионами.

К средствам из арсенала «нано», в соответствии с нанотехнологическим принципом «снизу - вверх», можно отнести приемы изменения состояния поверхности твердых частиц дисперсной фазы при введении добавок электролитов и ПАВ, а также пространственно-геометрической и энергетической обстановки в конденсационном объеме путем введения добавок-модификаторов вязкости и наномодификаторов.

\section{Заключение}

Прикладные инженерные решения по управлению реологией, структурообразованием и свойствами смесей и композитов для $3 \mathrm{D}-п е-$ чати должны быть основаны на применении традиционных рецептурнотехнологических факторов и использовании средств из арсенала «нано» . Управление требуется рассматривать одновременно как действие и процесс, а его результат оценивать по задаваемым реологическим свойствам смесей, параметрам кинетики схватывания и твердения, а также по достигаемому уровню качества композитов по конструкционным и функциональным характеристикам. Для проектирования номенклатуры эффективных составов смесей для 3D-печати и создания композитов различного функционального назначения необходимо решение следующих задач исследований: 1) изучение моделей реологического поведения; 2) изучение процессов схватывания и твердения; 3) обоснование требований к реологическим характеристикам и оптимизация моделей реологического поведения; 4) обоснование требований к кинетике процессов структурообразования и твердения в соответствии со скоростью печати строительных объектов. 
Работа выполнена по проекту 7.10781.2018/11.12 по государственному заданию «Выполнение проектов для получения первичных научных результатов, обеспечивающих расширение участия подведолственных образовательных организаций в реализации Национальной технологической инищиативы".

Данная публикация подготовлена при научных консультациях д.т.н., проф., академика РААСН Е.М. Чернышова.

\section{Библиографический список:}

1. Lim S., Buswell R.A., Le T.T., et al. Developments in construction-scale additive manufacturing processes //Automation in Construction. 2012. - 21(1). - Pp. 262-268.

2. Gebler M., Schoot Uiterkamp A.J.M., \& Visser C. A global sustainability perspective on 3D printing technologies // Energy Policy. 2014. - 74(C). - Pp. 158-167.

3. Tay Y.W., Panda B., Paul S.C., et al. Processing and Properties of Construction Materials for 3D Printing // Materials Science Forum. 2016. - 861. - Pp. 177-181.

4. Lloret E., Shahabb A.R., Linus M., et al. Complex concrete structures Merging existing casting techniques with digital fabrication // Computer-Aided Design. 2015. V. 60. - Pp. 40-49.

5. Weng Y., Li M., Qian S., et al. Design 3D printing cementitious materials via Fuller Thompson theory and Marson-Percy model // Construction and Building Materials. 2018. - V. 163. - Pp. 600-610.

6. Roussel N. Steady and transient flow behaviour of fresh cement pastes // Cement and Concrete Research. 2005. - 35 (9). - Pp. 1656-1664.

7. Khalil N., Aouad G., El Cheikh K.\& Rémond S. Use of calcium sulfoaluminate cements for setting control of 3D-printing mortars // Construction and Building Materials. 2017. - V. 157. - Pp. 382-391.

8. Panda B., \& Tan M.J. Experimental study on mix proportion and fresh properties of fly ash based geopolymer for 3D concrete printing // Ceramics International. 2018. V. 44. Is. 9. - Pp. 10258-10265.

9. Paul S.C., Tay Y.W.D., Panda B., \& Tan M.J. Fresh and hardened properties of 3D printable cementitious materials for building and construction // Archives of Civil and Mechanical Engineering. 2018. - V. 18(1). - Pp. 311-319.

10. Van Zijl A., Chandra S., Tan M.J., et al. Properties of 3D Printable Concrete $/ / 2^{\text {nd }}$ International Conference on Progress in Additive Manufacturing, (Pro-AM 2016). - At Nanyang, Singapore, 2016. - Pp. 421-426.

11. Perrot A., Rangeard D.\& Pierre A. Structural built-up of cement-based materials used for 3D-printing extrusion techniques // Materials and Structures. - 2016. - V. 49. Pp. 1213-1220.

12. Chernyshev E.M., Artamonova O.V., Slavcheva G.S. Concepts and technology base nano-modification of structures of building composites. Part 3: effective nanomodifica- 
tion of systems and structures of cement hardening cement stone (criteria and conditions) // Строительные материалы. - 2015. - № 10. - С. 54-63.

13. Чернышов E.M., Арталонова О.В., Славчева Г.С. Наномодифицирование систем твердения в структуре строительных композитов: монография. - Воронеж: Научная книга, 2016. - 132 с.

14. Чернышов E.M., Славчева Г.С., Артамонова О.В. Управление реологическими и конструкционными свойствами цементного камня при наномодифицировании // Нанотехнологии в строительстве. - 2016. - Том 8, № 6. - C. 87 - 101. - DOI: dx.doi.org/10.15828/2075-8545-2016-8-6-87-101.

15. Ребиндер П.А. Избранные труды. Поверхностные явления в дисперсных системах. Физико-химическая механика. - Москва: Наука, 1979. - 381 с.

16. Круглицкий Н.Н. Очерки по физико-химической механике. - Киев: Наукова думка, 1988. - $224 \mathrm{c.}$

17. Урьев Н.Б. Физико-химическая динамика структурированных нанодисперсных систем и нанодисперсных композиционных материалов. Часть 1 / Физикохимия поверхности и защита материалов. - 2010. - Т. 46, № 1. - С. 3 - 23.

18. Урьев Н.Б. Высококонцентрированные дисперсные системы. - Москва: Химия, 1980. -319 c.

19. Я Ялинский В.В., Пчелин В.А., Алелина Е.А., Щукин Е.Д. Коагуляционные контакты в дисперсных системах. - Москва: Химия, 1982. - 185 с.

20. Koch C.C. Nanostructured materials: Processing, Properties and Potential Applications. - New York: Noyes Publications, 2002. - 612 p.

\section{УВАЖАЕМЫЕ КОЛЛЕГИ!}

ПРИ ИСПОЛЬЗОВАНИИ МАТЕРИАЛА ДАННОЙ СТАТЬИ

ПРОСИМ ДЕЛАТЬ БИБЛИОГРАФИЧЕСКУЮ ССЫЛКУ НА НЕЁ:

Славчева Г.С., Арталонова О.В. Реологическое поведение дисперсных систем

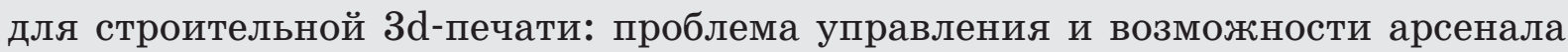
«нано» // Нанотехнологии в строительстве. - 2018. - Том 10, № 3. - С. 107122. - DOI: dx.doi.org/10.15828/2075-8545-2018-10-3-107-122.

\section{DeAr COlleagues! \\ THE REFERENCE TO THIS PAPER HAS THE FOLLOWING CITATION FORMAT:}

Slavcheva G.S., Artamonova O.V. The rheological behavior of disperse systems for $3 \mathrm{D}$ printing in constrcution: the problem of control and possibility of «nano" tools application. Nanotehnologii v stroitel'stve $=$ Nanotechnologies in Construction 2018, Vol. 10, no. 3, pp. 107-122. DOI: dx.doi.org/10.15828/2075-8545-201810-3-107-122. (In Russian). 